\title{
Darmtuberkulose als Hauptmanifestation einer systemischen Tuberkulose
}

\section{Gastrointestinal Tuberculosis as the Main Manifestation of Systemic Tuberculosis}

Autoren

Institute
H. Frickmann ${ }^{1 *}$, S. Jungblut ${ }^{1 *}$, H. Motherby ${ }^{2}$, J. Bargon

Medizinische Klinik, St. Elisabethen Krankenhaus, Katharina Kasper Kliniken, Frankfurt am Main

Gemeinschaftspraxis für Pathologie, Frankfurt am Main (S. Falk) eingereicht 3.62006

akzeptiert 13.72006

\section{Bibliografie}

DOI $10.1055 / \mathrm{s}-2006-944271$

Pneumologie 2006; 60; 667-671

(c) Georg Thieme Verlag KG

Stuttgart · New York

ISSN 0934-8387

Korrespondenzadresse PD Dr. Joachim Bargon Medizinische Klinik St. Elisabethen Krankenhaus Ginnheimer Straße 3 60487 Frankfurt/Main bargon@em.uni-frankfurt.de

\section{Zusammenfassung}

Wir beschreiben den seltenen Fall einer Tuberkulose mit überwiegend gastrointestinaler Symptomatik. Die 52-jährige Patientin stellte sich mit unklaren Unterbauchbeschwerden und therapierefraktärem Aszites vor. Im CT des Thorax fanden sich rechtsbetont konfluierende Lymphknoten, im CT des Abdomens neben Aszites noduläre Strukturen in Zökumnähe. Im Rahmen von Koloskopie, diagnostischer Laparoskopie und Bronchoskopie konnten Biopsien aus der Kolonmukosa, dem entzündlich veränderten Peritoneum, dem linken Oberlappenbronchus und den mediastinalen Lymphknotenpaketen entnommen werden. In den peritonealen Biopsien waren histologisch Granulome mit verkäsenden Nekrosen nachweisbar, in den mediastinalen Lymphknotenbiopsien Mycobacterium tuberculosis in der PCR. Ferner gelang im Aszitespunktat in der Kultur die Anzucht von Mycobacterium tuberculosis. Unter Therapie mit Isoniazid, Rifampicin, Ethambutol und Pyrazinamid kam es zur Besserung von Symptomatik und Befund.

\section{Einleitung}

Wir beschreiben einen der seltenen Fälle einer Dickdarmtuberkulose im Bereich des Zökums. Wenngleich die Tuberkulose vor allem in Entwicklungsländern endemisch beheimatet ist, führen Migrationsbewegungen und die Ausbreitung der Immunschwächekrankheit AIDS zu einem Wiederanstieg der Inzidenz auch in den europäischen Ländern [1]. In Europa wurde eine jährliche Inzidenzzunahme von $0,4 \%$ beobachtet [2]. In sehr seltenen Fällen manifestiert sich die Krankheit als Abdominaltuberkulose. Aufgrund der oft unspezifischen Symptomatik kann die Diagnose dann erschwert sein [1].

\section{Abstract}

We describe a rare case of tuberculosis with mainly gastrointestinal problems. The 52-yearaged female patient came to hospital with unclear pain in the lower abdomen and ascites that was refractory to therapy. The computed tomography of the thorax showed right-sided confluating lymphoid nodes, the CT of the abdomen showed ascites and nodular structures near the coecum. Tissue samples were taken from the mucosa of the colon, the inflammatory altered peritoneum, the left bronchus of the upper lobe and the confluating lymphoid nodes in the mediastinum during colonoscopy, diagnostic laparoscopy and bronchoscopy. The samples from the peritoneum showed granulomas with caseating necroses in histological slices. Mycobacterium tuberculosis was detected by PCR in the tissue samples from the lymphoid tissue of the mediastinum. Furthermore, Mycobacterium tuberculosis grew in cultures from samples of the abdominal ascites. The symptoms and pathological findings improved under a therapy comprising isoniazid, rifampicin, ethambutol and pyrazinamid.

\section{Anamnese und körperliche Untersuchung}

Die marokkanische 52-jährige adipöse Patientin stellte sich mit passageren Bauchschmerzen und progredientem Aszites im rechten Unterbauch in reduziertem Allgemeinzustand vor. Übelkeit, Erbrechen, Teerstuhl und Gewichtsverlust wurden verneint. Nachdem sich die Patientin zunächst in gynäkologische Behandlung begeben hatte, wurde sie mit deutlichem Aszites in die internis-

\footnotetext{
Hagen Frickmann und Sven Jungblut sind gleichermaßen als Erstautoren anzusehen.
} 
tische Abteilung überwiesen. Anamnestisch waren vor 6 Jahren Lymphome im Bereich beider Tracheobronchialwinkel als $\mathrm{Zu}-$ fallsbefund entdeckt worden, wobei damals histologisch die Diagnose einer epitheloidzellig granulomatösen Lymphadenopathie im Sinne einer Sarkoidose gestellt wurde. Alkohol- und Nikotinabusus wurden verneint.

Die Patientin gab an, zuletzt vor 2 Jahren in Marokko gewesen zu sein. Vor zwei Monaten sei es nach dem Genuss von Feigen zu heftigen abdominalen Schmerzen und Durchfall gekommen. Die Farbe des Stuhls war nicht verändert. Im familiären Umfeld der als Hausfrau lebenden Patientin waren keine Fälle von Tuberkulose bekannt.

Mit Ausnahme einer grenzwertigen Tachykardie von 100/min und einem beidseitigen Druckschmerz im Unterbauch gestaltete sich die körperliche Untersuchung unauffällig.

\section{Pathologische Laborwerte}

Bei Aufnahme lag ein CRP-Wert von 52,6 MG/L (Referenz 0-5 MG/L) vor, der sich nach wenigen Tagen auf maximal 82,5 MG/L steigerte, die Blutsenkung war mit 54/70 erhöht. Darüber hinaus waren die Blutwerte unauffällig.

Stuhluntersuchungen ergaben kein Wachstum von Salmonellen, Shigellen, Yersinien, Campylobacter oder anderen darmpathogenen Keimen, die Serologie auf HIV, Yersinien und Amöben und der Test auf Clostridium-difficile-Toxin verliefen negativ.

\section{Sonographie}

Neben einem massiven Aszites ( $\mathbf{A b b} \mathbf{1}$ ) fanden sich in der Sonographie des Abdomens im rechten Mittel- und Unterbauch mehrere echoreiche Raumforderungen im Sinne vergrößerter mesenterialer Lymphknoten. Im Aszitespunktat imponierten zahlreiche neutrophile Granulozyten und Lymphozyten, monozytoide Zellen, Mesothelien und Erythrozyten, jedoch ergab sich kein Anhalt für Malignität. Säurefeste Stäbchen wurden nicht nachgewiesen, in der angelegten Kultur wuchs Mycobacterium tuberculosis mit Empfindlichkeit auf alle erstrangigen $\mathrm{Tu}-$ berkulostatika.

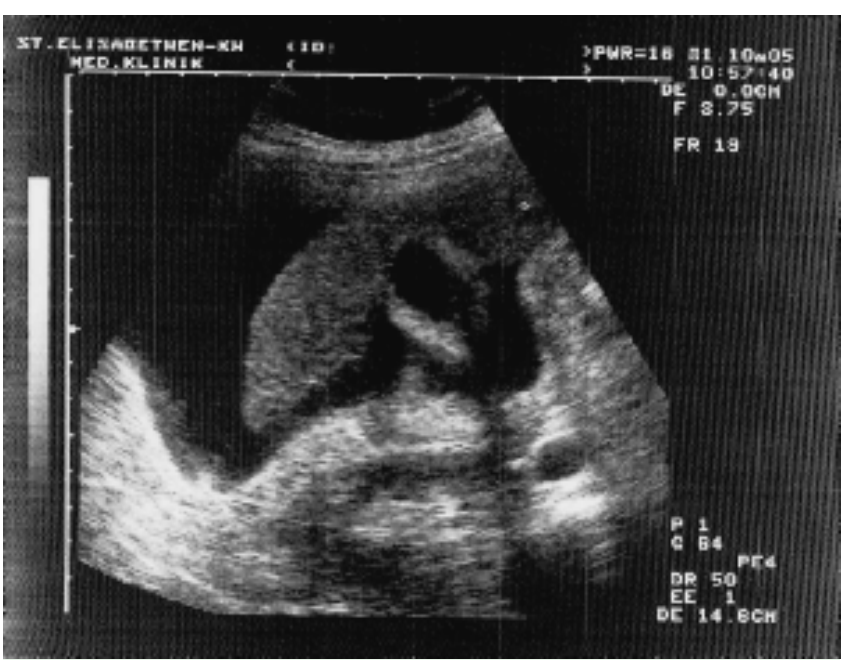

Abb. 1 Sonographisch massiver Aszites im Abdomen.

\section{Röntgen und Computertomographie}

Der Röntgenbefund des Thorax war altersentsprechend unauffällig. Im nativen und mit Kontrastmittel durchgeführten CT des Thorax fanden sich rechtsbetont multiple, zu einem Paket mit einem maximalen Durchmesser von $4 \mathrm{~cm}$ konfluierende Lymphknoten, die vom oberen Mediastinum bis infracarinal reichten ( Abb.3). Hinweise für einen intrapulmonalen spezifischen Prozess oder einen Erguss ergaben sich nicht. Im CT des Abdomens zeigten sich neben deutlichem Aszites multiple noduläre weichteildichte Strukturen, gruppiert in Zökumnähe ( $\mathbf{A b b . 2}$ ).

\section{Gastroskopie}

Es zeigten sich eine kleine Hiatushernie ohne Refluxzeichen und eine vergröberte Schleimhaut mit punktförmiger Rötung im Corpusanteil des Magens. Biopsien ergaben eine chronisch gering aktive Gastritis des Corpus und Antrum ventriculi mit Regenerationszeichen, einer intestinalen Metaplasie und einer Schleimhauthyperämie.

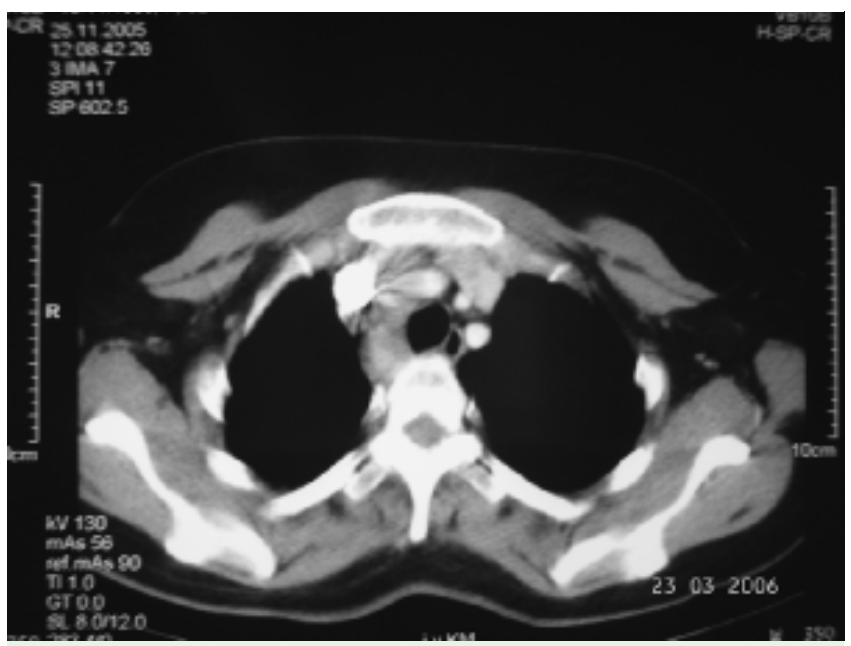

Abb. 2 Konfluierende mediastinale Lymphknoten im CT des Thorax.

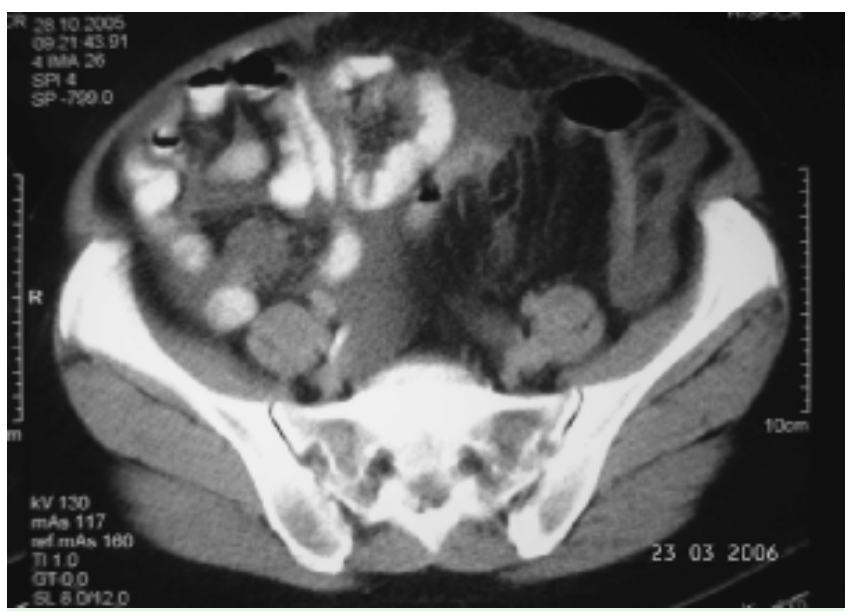

Abb. 3 Noduläre, weichteildichte Strukturen in Zökumnähe im CT des Abdomens. 


\section{Koloskopie}

Bei sonst unauffälligem Dickdarm wurden im Bereich der Ileozökalklappe und des Zökalpols multiple polypöse und tumoröse, z.T. ulzerierende Veränderungen bei verquollen adenoid imponierender Mukosa entdeckt. Eine Passage der Bauhin'schen Klappe mit endoskopischem Vordringen in das terminale Ileum war nicht möglich. Probebiopsien aus den Bereichen der Schleimhautanomalien des Zökums zeigten eine ulzeröse Kolitis mit regeneratorischen Epithelveränderungen im Sinne einer chronisch entzündlichen Darmerkrankung. Die Kolonschleimhaut imponierte mit gestörter Kryptenarchitektur, ferner lag ein mäßiges Schleimhautödem mit reichlich dilatierten, erythrozytengefüllten Blutgefäßen sowie einem Infiltrat aus Lymphozyten, Plasmazellen sowie vereinzelt neutrophilen und eosinophilen Granulozyten vor. Epitheliale Dysplasien oder ein Anhalt für Malignität fanden sich nicht.

\section{Diagnostische Laparoskopie}

Im Bereich des Zökums imponierte eine ausgeprägte granulomatöse Entzündungsreaktion, die das gesamte Peritoneum mit einbezog ( Abb.4). Entnommene Gewebeproben ergaben eine granulomatöse Entzündung unter Ausbildung unterschiedlich großer Granulome mit Riesenzellen vom Langhans-Typ und stellenweise kleinherdigen Nekrosen ( Abb.5). Säurefeste Stäbchen waren darin nicht nachweisbar.

\section{Endosonographie und Bronchoskopie}

Unter endosonographischer Kontrolle wurden die im CT diagnostizierten konfluierenden Lymphknoten des Mediastinums zur Biopsieentnahme punktiert. In den Punktaten wurde mittels der Polymerasekettenreaktion (PCR) Mycobacterium tuberculosis nachgewiesen. Die histologische Aufarbeitung des Lymphknotengewebes ergab stark regressiv verändertes, überwiegend von Makrophagen infiltriertes Zellmaterial. Biopsien aus dem linken Oberlappenbronchus zeigten eine Schleimhautfibrose mit entzündlicher Infiltration der teilfibrosierten Lamina pro-

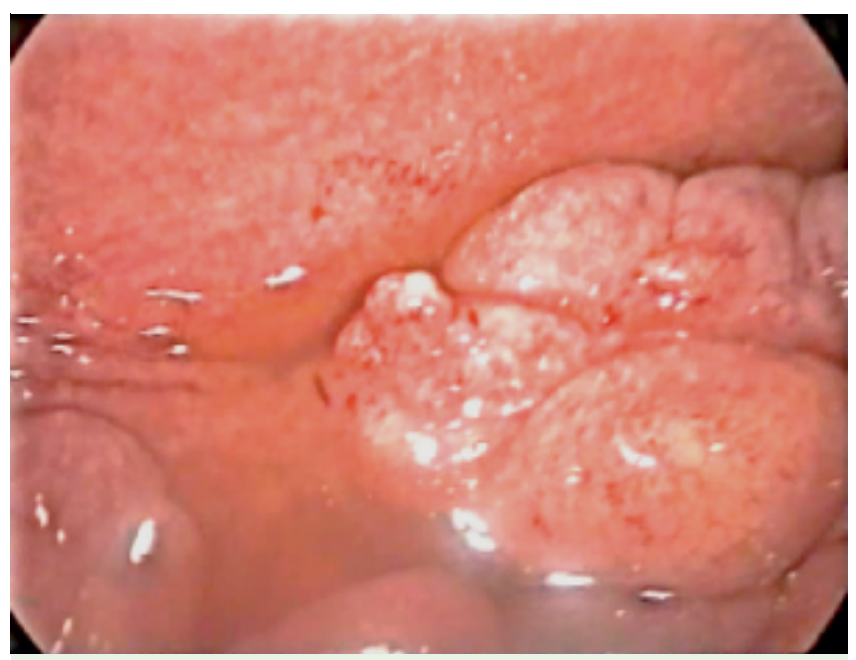

Abb. 4 Peritoneale granulomatöse Entzündung als laparoskopischer Befund. pria bei regelrechtem mehrreihigen Zylinderepithel an der Oberfläche. Im Bronchialsekret und Sputum war kein pathologischer Befund zu entdecken. Die bronchoalveoläre Lavage ergab eine normale Gesamtzellzahl bei relativer Lymphozytose und erniedrigter CD4/CD8-Ratio: In $50 \mathrm{ml}$ 5,5 Millionen Zellen insgesamt, Makrophagen 44\%, Lymphozyten 53\%, Neutrophile 1\%, Eosinophile $2 \%$, CD4/CD8-Ratio 0,42.

\section{Therapie und Verlauf}

Eine tuberkulostatische Vierfachtherapie mit Isoniazid, Rifampicin, Ethambutol und Pyrazinamid wurde eingeleitet. Die chronische Gastritis wurde mit Esomeprazol behandelt. Der Aszites wurde mit Furosemid ausgeschwemmt.

Innerhalb von 4 Wochen kam es zu einer Besserung der Beschwerdesymptomatik. Eine Kontrollkoloskopie zeigte einen deutlich rückläufigen Lokalbefund. Die Schleimhaut imponierte noch immer adenoid verquollen, die anatomischen Strukturen des Zökums und des terminalen Ileums waren jedoch gut zu differenzieren und mit dem Endoskop frei passierbar.

Die Patientin wurde zur strengen Fortführung der tuberkulostatischen Therapie angehalten und in gutem Allgemeinzustand entlassen. Eine Kontrollkoloskopie nach zwei Monaten wurde vereinbart.

\section{Diskussion}

Obwohl sich die Tuberkulose in den meisten Fälle in der Lunge manifestiert, kann die Darmtuberkulose als Bestandteil eines Multiorganbefalls oder als primäre gastrointestinale Tuberkulose auftreten. Während bis zu 40\% der Patienten mit Darmtuberkulose an AIDS leiden, stellen auch Alkoholabusus, intravenöser Drogenabusus, chronischer Steroidabusus, Proteinmalnutrition und hohes Alter Risikofaktoren dar [3]. Unsere Patientin ließ sich anamnestisch keiner entsprechenden Risikogruppe zuordnen. Auffällig war jedoch bei der Patientin die Anamnese der 6 Jahre zuvor diagnostizierten epitheloidzellig granulomatösen Lymphadenopathie im Bereich der Tracheobronchialwinkel. Man könnte spekulieren, dass damals eine Lymphknotentuberkulose vorlag und die jetzige Erkrankung als postprimäre Tuberkulose einzuschätzen ist.

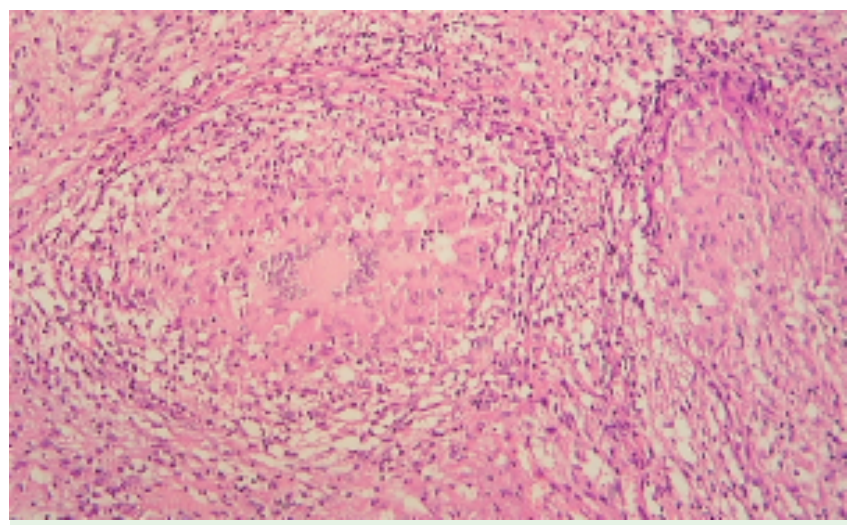

Abb. 5 Peritoneale Probeexzision mit einer granulomatösen Entzündungsreaktion entsprechend dem Bild einer Tuberkulose mit epitheloidzelligen Granulomen und umgebender Entzündungsreaktion (HE-Färbung, $100 \times$ Vergrößerung). 
Die Hauptlokalisation der gastrointestinalen Tuberkulose ist der Bereich des terminalen Ileums und der Ileozökalregion, nur sehr selten ist der gesamte Gastrointestinaltrakt befallen. Der häufige Befall der Ileozökalregion resultiert aus der dortigen relativen physiologischen Stase bei hoher Absorptionsrate, vollständigerer Digestion und reichlich vorhandenem lymphatischen Gewebe [4]. Auch in unserem Fall trat die Darmtuberkulose an ihrer typischen Lokalisation auf.

Der tuberkulösen Enteritis können die hämatogene Aussaat einer aktiven Lungen- oder Miliartuberkulose, das Verschlucken von infektiösem Sputum durch Patienten mit aktiver Lungentuberkulose, die Ingestion von kontaminierten Nahrungsmitteln wie z. B. von Mycobacterium-bovis-haltiger Milch und die kontinuierliche Ausbreitung von Nachbarorganen zugrunde liegen [5].

Die Symptomatik ist meist unspezifisch. Das Hauptsymptom ist der abdominelle Schmerz, darüber hinaus kann es zu Verstopfungen oder Diarrhöen, Gewichtsverlust, Anorexie, Veränderungen der Essgewohnheiten, Malabsorption, Fieber mit Nachtschweiß, Nausea, Vomitus, Meläna und rektalen Blutungen kommen [6]. Der von uns beschriebene Fall macht deutlich, dass die Symptomatik jedoch auch ausgesprochen blande sein kann.

In der körperlichen Untersuchung imponiert der Bauch meist weich, eine Raumforderung im rechten unteren Quadranten ist in einem Viertel bis der Hälfe der Fälle tastbar [4]. Gelegentlich tritt ein Ileus auf.

Die radiologische bildgebende Diagnostik ist meist unspezifisch. Die Computertomographie hat eine Sensitivität von etwa 67\%, wobei Adenopathie, Splenomegalie, Hepatomegalie und Aszites zu den auffälligen Befunden gehören [7]. In dem von uns beschriebenen Fall sah man neben dem Aszites lediglich unspezifische noduläre Strukturen.

Die definitive Diagnose kann durch typische Histologie, Kultur und Ziehl-Neelsen-Färbung auf säurefeste Stäbchen gestellt werden. Gastroskopie und Koloskopie sind die diagnostischen Methoden der Wahl, um die durch die gastrointestinale Tuberkulose induzierten Veränderungen am Gastrointestinaltrakt zu beurteilen. Klinisch werden die Läsionen in ein akutes Stadium mit Ulzerationen und ein chronisch hypertrophisches Stadium mit Granulomen und starker Fibrose eingeteilt. Die Ulzerationen imponieren typischerweise mit einer irregulären Form und einem nekrotischen Ulkusgrund, was zu Perforationen und Fisteln führen kann [8]. Typische Granulome können bei der gastrointestinalen Tuberkulose lediglich in $18-41 \%$ der Fälle histologisch dargestellt werden. Gelegentlich treten sie in der Submukosa auf, was bei zu flacher Biopsieentnahme die Diagnose erschweren kann $[9,10]$. Bei nur etwa $40 \%$ der Granulome im Dickdarm ist Verkäsung zu beobachten [11]!

Eine mikrobiologische Analyse ist nur von begrenztem Wert bei der Diagnosefindung. Färbungen auf säurefeste Stäbchen sind in Kolonbiopsien nur in $32-35 \%$ positiv, die Kultur gelingt nur in 36 - 40\% der Fälle [9,12]. Die PCR-Analyse auf Mycobacterium tuberculosis soll in 60\% der Kolonbiopsien positiv und innerhalb von 48 Stunden auswertbar sein [13], was sie bei Verdacht zur Methode der Wahl macht.

In Fällen, in denen PCR und Kultur zu negativen Ergebnissen führen, kann die Diagnose histologisch und aufgrund des Ansprechens auf die tuberkulostatische Therapie gestellt werden [14]. Im von uns beschriebenen Fall wurde die gastrointestinale Tuberkulose letztlich aus der Zusammenschau von Bildgebung, Histologie, Kultur und PCR diagnostiziert.
Zur Diagnosesicherung einer abdominalen Tuberkulose kann die Laparoskopie indiziert sein, zumal diese Krankheit als häufige Manifestation - wie in unserem Fall - eine tuberkulöse Peritonitis aufweist [15].

Mögliche Differenzialdiagnosen der gastrointestinalen Tuberkulose sind Morbus Crohn, Lymphome, maligne Neoplasien wie Sarkome, Divertikulitis, Appendizitis mit periappendikalem Abszess und andere Infektionskrankheiten des Gastrointestinaltrakts wie Amöbiasis und Yersiniose [8]. In der Differenzierung zwischen einer Darmtuberkulose und chronisch entzündlichen Darmerkrankungen wie Morbus Crohn ist die alleinige Koloskopie nur von begrenzter Aussagekraft, da beide Krankheitsentitäten unter einem sehr ähnlichen Bild mit diskontinuierlichem Befall, Stenosen und sogar Fisteln imponieren können [10]. An die Möglichkeit der Darmtuberkulose muss aber gedacht werden. Therapie der Wahl ist eine Kombinationstherapie aus Isoniazid, Rifampicin, Ethambutol und Pyrazinamid. Bei bestehenden Resistenzen kann auch Ciprofloxacin zum Einsatz kommen. Bei histologisch gesichertem Verdacht sollte die Therapie bereits vor Eintreffen der Ergebnisse aus der Kultur eingeleitet werden, da die Erkrankung - besonders bei immuninkompetenten Patienten - unbehandelt rapide progredient und tödlich verlaufen kann [16]. Zu den Indikationen, bei denen eine operative Therapie nötig wird, gehören Obstruktionen, Fistelbildungen, Perforationen und größere Blutungen [17].

Die gastrointestinale Tuberkulose sollte als mögliche, wenn auch seltene Differenzialdiagnose unklarer gastrointestinaler Beschwerden berücksichtigt werden. Bei früher Diagnose und therapeutischer Intervention ist sie mit guter Prognose behandelbar, wie der von uns beschriebene Fall veranschaulicht. Unter tuberkulostatischer Vierfachtherapie kam es bei unserer Patientin zu einem deutlichen Rückgang von Symptomatik und Befund, so dass sie das Krankenhaus in gutem Allgemeinzustand verlassen konnte.

Der allgemeinen Therapieempfehlung einer Kombinationstherapie aus Isoniazid, Rifampicin, Ethambutol und Pyrazinamid für die Behandlung der gastrointestinalen Tuberkulose [16] können wir uns aufgrund des guten Ansprechens unserer Patientin auf dieses Therapieregime nur anschließen. Ein ergänzender Einsatz von Ciprofloxacin war in unserem Fall nicht nötig, ebenso wenig wie eine operative Therapie, die oben genannten Indikationen vorbehalten bleibt. Dennoch bestätigt dieser Fall die Sinnhaftigkeit einer laparoskopischen Intervention zur Diagnosesicherung. Zudem demonstriert er eindrucksvoll die meist gute Prognose der gastrointestinalen Tuberkulose bei konsequenter Therapie.

\section{Literatur}

1 Gerhardt T, Wolff M, Fischer HP et al. Pitfalls in the diagnosis of intestinal tuberculosis: A case report. Scandinavian Journal of Gastroenterology 2005; 40: 240-243

2 Haas W, Altmann D. Zur Situation bei wichtigen Infektionskrankheiten in Deutschland: Tuberkulose im Jahr 2002. Epidemiologisches Bulletin 2003; 50: 419-420

3 Settbas Y, Alper M, Akcan Y et al. Massive gastrointestinal tuberculosis in a young patient without immunosuppression. World J Gastroenterol 2003; 9: $2382-2384$

4 Horvath KD, Whelan RL. Intestinal Tuberculosis: Return of an old disease. Am J Gastroenterol 1998; 93: 692 -696

5 McGee GS, Williams LF, Potts J et al. Gastrointestinal tuberculosis resurgence of an old pathogen. Am Surg 1989; 55: 16 - 20

6 Brizi MG, Celi G, Scaldazza AV et al. Diagnostic imaging of abdominal tuberculosis: gastrointestinal tract, peritoneum, lymph nodes. Rays 1998; 23: $115-125$

7 Hulnick DH, Megibow AJ, Naidich DP et al. Abdominal tuberculosis. CT evaluation. Radiology 1985; 157: 1999-2004 
8 Tabrisky J, Lindstrom RR, Peters R et al. Tuberculous enteritis. Review of a protean disease. Am J Gastroenterol 1975; 63: 49-57

9 Bhargava DK, Kushwaha AK, Dasarathy S et al. Endoscopic diagnosis of segmental colonic tuberculosis. Gastrointest Endosc 1992; 38: 571 574

10 Marshall JB. Tuberculosis of the gastrointestinal tract and peritoneum. Am J Gastroenterol 1993; 88: 989 - 999

11 Kochhar R, Rajwanshi A, Goenka MK et al. Colonoscopic fine needle aspiration cytology in the diagnosis of ileocoecal tuberculosis. Am J Gastroenterol 1991; 86: 102 - 104

12 Kim KM, Lee A, Choi KY et al. Intestinal tuberculosis: clinicopathologic analysis and diagnosis by endoscopic biopsy. Am J Gastroenterol 1998; 93: 606-609
13 Anand BS, Schneider FE, El-Zaatari FA et al. Diagnosis of intestinal tuberculosis by polymerase chain reaction on endoscopic biopsy specimens. Am J Gastroenterol 1994; 89: 2248 - 2249

14 Artu P, Lavergne-Slove A, Joly F et al. Isolated jejunal tuberculosis mimicking Crohn disease. Diagnosis by push video-enteroscopy. Gastroenterol Clin Biol Biol 1999; 23: 1086 - 1089

15 Ibrarullah M, Mohan A, Sarkari A et al. Abdominal tuberculosis: diagnosis by laparoscopy and colonoscopy. Trop Gastroenterol 2002; 23 : $150-153$

16 Berning SE. The role of fluoroquinolones in tuberculosis today. Drugs 2001; 61: 9-18

17 Vaidya MG, Sodhi JS. Gastrointestinal tract tuberculosis: a study of 102 cases including 55 hemicolectomies. Clin Radiol 1978; 29: 189-195 